Aus dem Institut für radiologische Diagnostik und Therapie im k. k. allgemeinen Krankenhaus in Wien.

\title{
Die Duodenalstenose durch Füllung und Peristaltik radiologisch erkennbar.
}

\author{
Von Privatdozent Dr. G. Holzknecht, Leiter des Instituts.
}

(Mit 9 Abbildungen.)

Die Literatur kennt keine radiologischen Symptome der Duodenalstenose und weist auch keinen Fall von Duodenalstenose auf, welcher radiologisch verfolgt worden wäre. Die Bemühungen, die Symptomatologie nach dieser Richtung zu erweitern, waren sehr wünschenswert, weil die klinische Diagnostik dieser Störung über Vermutungen selten hinauskommt. Nur in den fortgeschrittensten und ausgesprochensten Fällen kann sie sicher gestellt werden. Sie ist wahrscheinlich, wenn sie durch retrograde Fortsetzung der Stauung in den Magen und Erlahmung der kompensatorischen Mehrleistung auch dieses Organes jene Symptome hervorruft, welche sonst durch die Pylorusstenose erzeugt werden, und wenn dabei regelmäßig Galle im Erbrochenen oder Mageninhalt gefunden wird. Wenn aber Steifung und Peristaltik bei noch nicht abgemagerten Kranken fehlen, so kann das kopiöse Erbrechen galliger Massen auch durch Hypersekretion hervorgerufen werden. Tatsächlich war in keinem meiner Fälle vor der Röntgenuntersuchung an Duodenalstenose gedacht worden, und nur bei einem wurde nachträglich der Regelmäßigkeit des galligen Erbrechens Beachtung geschenkt und so auch, abgesehen von der Röntgenuntersuchung, die Diagnose gesichert. Die $\mathrm{Zu}$ weisungen stellten vielmehr meist Pylorusstenose oder Gastrektasie in engere Frage.

Das späte Auftreten der Koliken post coenam hat sich als Duodenalstenosensymptom nicht nur praktisch nicht bewährt. Die Röntgenuntersuchung an Normalen 
Die Duodenalstenose durch Füllung und Peristaltik radiologisch erkennbar. 55

hat auch theoretisch eine Tatsache ergeben, welche diesem Symptom den Boden entzieht. Nach der Nahrungsaufnahme in den Magen bleibt der Pylorus nämlich nicht lange Zeit geschlossen, und die ersten Ingesten gelangen nicht spät ins Duodenum, so daß am Magen eine besondere Misch- und Austreibungsperiode unterschieden werden könnte, sondern die Austreibung geeigneter Teile der Mahlzeit beginnt sofort nach der Einnahme. Mischung und Austreibung laufen nebeneinander her. Wenn ,,das spätere Auftreten der Duodenalkolik" überhaupt richtiger Beobachtung sein Entstehen verdankt, so muß man wohl den Grund dafür in den gleichen, noch unbekannten Momenten sehen, welche bewirken, daß auch die Stenosenkoliken des Magens nicht zugleich mit der Austreibungsarbeit des Magens einsetzen, sondern erst längere Zeit nach deren Beginn.

Solcherart müssen die Konsequenzen der Duodenalstenose noch weiter gediehen sein, als die der Pylorusstenose, wenn sie erkennbar sein soll.

Sie ist unerkennbar im ersten Stadium, dem der Kompensation. Solange der dahinterliegende Duodenalabschnitt und der Magen zusammen genommen durch Hypertrophie und Mehrleistung das Hindernis in erträglichen Zeiten zu überwinden vermögen, bewirkt die Duodenalstenose nur eine diagnostisch unzuverlässige Erscheinung, die Kolikanfälle, während das gleiche Stadium bei der Pylorusstenose schon in der Steifung und der Peristaltik charakteristische Lokal-und Organsymp t o m e besitzt. Solche zeigt bei Anwendung der älteren Untersuchungsmethoden die Duodenalstenose nie. Weder die abnorme Weite des Organes noch seine abnorm lang bestehende Füllung, noch auch seine tonische und peristaltische Kontraktion sind für jene zugänglich, und der Grund dafür ist seine tiefe, unzugängliche Lage und Fixation an der hinteren Abdominalwand, dem Pankreas benachbart, dessen Veränderungen uns aus dem gleichen Grunde fast stets entgehen.

Nun könnte noch mit einem Schein von Berechtigung argumentiert werden, da $B$ es praktisch gleichgültig sei, ob die Diagnose auf Pylorus-oder Duodenalst e nos e fällt, nur die Sicherheit, daß eine von beiden besteht, kommt in Betracht. Denn die Therapie, die fast stets in der 
Gastroenterostomie bestehen wird, sei für beide die gleiche.

Dazu ist zunächst $\mathrm{zu}$ wiederholen, daß die Duodenalstenose naturgemäß in vielen Fällen (besonders den tiefsitzenden) niemals, immer aber viel später die Erscheinungen der Pylorusstenose macht, als diese selbst, so daß sie auch viel später oder nie Anlaß zur Gastrostomie gibt, und daß daher ihre Symptomatologie auch rein praktisch wichtig ist. Die Mehrzahl unserer Fälle wurden ja nicht einmal als Pylorusstenosen zur weiteren Aufklärung, z. B. über die Natur derselben zugewiesen, sondern nur mit dem Verdacht auf eine Stenose des Pylorus, einige boten sogar für diesen keine Anhaltspunkte.

Ferner scheint mir die Gastroenterostomie allein keineswegs die Operation der Wahl zu sein, weil sie ohne Not die physiologischen Leistungen der Pars pylorica, des Pylorus ausschaltet und die des Duodenum mehr oder minder erheblich reduziert. Wenigstens bei tiefem Sitz der Duodenalstenose mußte man der Galle und dem Succus pankreaticus den Umweg über den Magen um so mehr ersparen und die Auslesefunktion des Pylorus um so mehr erhalten, als die tiefe Duodenojejunostomie die Gastroenteroanastomose nicht allzusehr an Schwierigkeit übertreffen dürfte.

Endlich fällt noch ins Gewicht, daß es leicht geschehen kann, daß bei der Laparotomie unter Annahme einer Pylorusstenose, wenn der Pylorus frei gefunden wird, sogar von der Gastrostomie ganz abgesehen wird, ein Fall, der geeignet ist, das so verbreitete Vederemo probelaparotomiefreudiger Chirurgen ad absurdum zur führen.

Kann man den bisherigen Nachweis der Duodenalstenose als unsicher und spät bezeichnen, so muß der radiologische leicht genannt werden. Die Ursache davon liegt in der ziemlich guten Zugänglichkeit des Duodenum für die Röntgendurchleuchtung, für die auch die tiefe Lage kein nennenswertes Hindernis ist.

Radiologisch sichtbar sind: der Eintritt der schweren stark absorbierenden Ingesten ins Duodenum, a u chleinerer Partikel, ihr Verweilen, ihre Anhäufung, seine Füllung, Länge, Weite, sein Verlauf, Krümmung bzw. 
Die Duodenalstenose durch Füllung und Peristaltik radiologisch erkennbar. 57

Knickung, die Verweildauer, die Inhaltsbewegung und Weiterbeförderung in den D ünndarm, und bei genügender Füllung die die Inhaltsbeförderung begleitenden Formver änderungen.

Unter diesen Umständen konnte man von der Durchleuchtung vieles erwarten.

Tatsächlich habe ich schon vor 4 Jahren unter den ersten Magenuntersuchungen ein Kind mit den klinischen Zeichen der stenotischen Gastrektasie untersucht, das Erscheinungen bot, die ich nur durch eine Stenose in der Pars horizontalis inferior oder zwischen dieser und dem Jejunum verstehen konnte, welche der Anamnese nach angeboren schien. Es geriet mir in Vergessenheit, bis im Vorjahre wieder ein Fall gelegentlich der auf den Magen gerichteten Untersuchung beobachtet wurde. Nun kamen bis Ende des Jahres weitere 3 Fälle vor und im Januar dieses Jahres wieder einer, der in dieser Arbeit nicht verwertet ist.

Daraus ergibt sich eine überraschende Häufigkeit der Duodenalstenose gegenüber der bisherigen Annahme, die eine Mitteilung um so mehr rechtfertigt, als der Nachweis recht leicht und sicher ist.

Mit der Publikation habe ich gewartet, weil ich von keinem eine autoptische Bestätigung erlangte. Aber die Sinnfälligkeit, Konstanz und Verständlichkeit des sich immer in gleicher Weise wiederholenden Symptomenbildes läßt nun keinen Zweifel mehr zu.

Das normaleradiologische Verhalten des $\mathrm{Duod}$ e n u m.

Die radiologisch sichtbaren Erscheinungen und Vorgänge am Duodenum, von denen diejenigen bei Duodenalstenose fast in jedem Punkte abweichen, sind in der bisherigen Literatur nur unvollständig und in unzusammenhängenden Andeutungen dargestellt. Es tut daher not, nicht nur das typische normale Verhalten, sondern auch die hier häufigen Varianten kennen zu lernen. Dazu bot die radiologische Untersuchung des Magens nach der vom Verfasser und seinen Mitarbeitern ausgearbeiteten 
Methode besonders günstige Verhältnisse, weil nur die Verwendung der Durchleuchtung durch kontinuierliches Verfolgen der Ingesten die Identität des gefüllten Dünndarmabschnittes mit dem Duodenum festzustellen erlaubt, weil nur sie den zeitlichen Ablauf, der Füllung und Durchwanderung und die übrigen peristaltischen Vorgänge zu beobachten erlaubt, während die photographischen Untersuchungen infolge der Un-

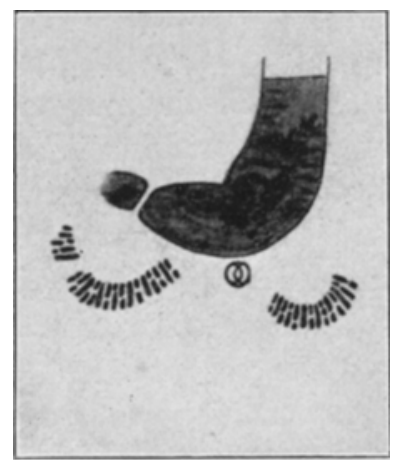

Fig. I.

Füllung des Magens und typisches Inhaltsbild des normalen Duodenums. Magen von Übergangsform (Fa ulhaber) zwischen Hackenmagen u. Stierhornmagen, ausgußartig gefüllt. Ebenso ist der Bulbus duodeni gefüllt. Zwischen beiden ein heller Spalt, der Muskelring des Pylorus. Im übrigen Duodenum zusammenhängende Wismutmengen, welche durch leichten Druck quergestreift erscheinen. Ebenso in der ersten Jejunalschlinge. vollständigkeit und Flüchtigkeit der Füllung nichts Wesentliches ergeben haben.

Durch diese Umstände haben einige tausend Magenuntersuchungen, die in meinem Institut ausgeführt wurden, Gelegenheit zur Beobachtung am Duodenum gegeben.

Ich fasse im folgenden kurz zusammen, was ich in der Mitteilung über, ,d as normale röntgologische Verhalten des Duoden u m s" (Zentralblatt für Physiologie IgIo) als Ergebnis der Untersuchungen dargestellt habe: Der flüssige Inhalt des Duodenums gelangt in dieses auf zweierlei Weise, erstens ohne Mithilfe der Magenperistaltik, wenn bei leerem Magen, daher offenem Pylorus, der Magenverdauung nicht bedürftiger Ingesten, z. B. Wismutwasseraufschwemmung, getrunken wird, und zwar ohne Aufenthalt im Magen, wenn der Pylorus an tiefster Stelle liegt. Das letztere ist der Fall beim normalen rinderhornförmigen Magen und aufrechter Körperstellung, ferner auch beim hakenförmigen, aber nur in rechter Seitenlage. Bei dieser Form des Magens kann das Ausfließen ins Duodenum in aufrechter Körperstellung durch manuelles Heben des Inhaltes bis zur Höhe des Pylorus (Effleurage ins 
Die Duodenalstenose durch Füllung und Peristaltik radiologisch erkennbar. 59

Duodenum) oder durch Anwendung von Flüssigkeit im Überschusse bewerkstelligt werden. Zweitens treibt der Magen mittels der Antrumperistaltik seinen flüssigen oder verflüssigten Inhalt rhythmisch in das Duodenum.

Hier sammelt sich das Ejakulat in der ersten Hälfte der Pars superior Duodeni, deren Weite und leichte Entfaltbarkeit die Kraft des Spritzstrahles bricht. Kleinere Mengen können hier lange Zeit liegen bleiben, vielleicht einer der Magenverdauung ähnlichen Nachverdauung unterworfen, worin der Befund von den Magendrüsen ähnlichen Drüsen eine ergänzende Erklärung

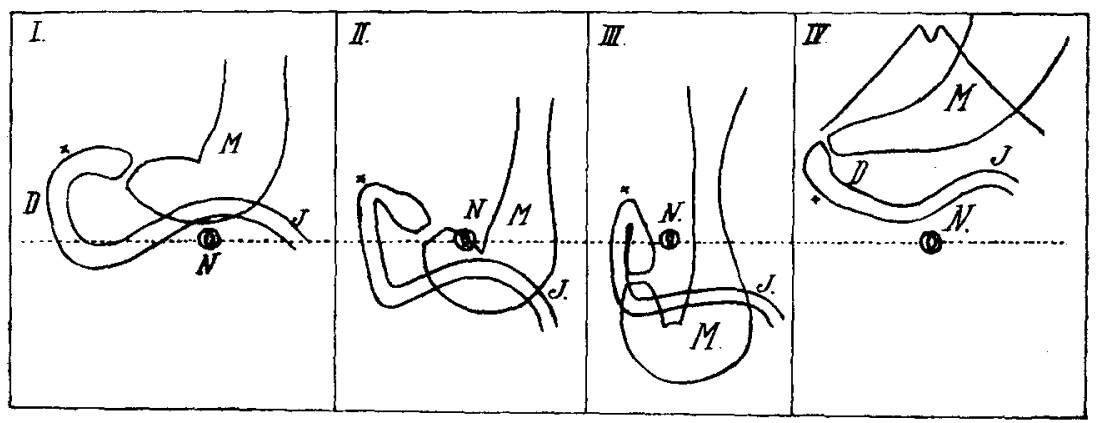

Fig. 2.

Zu Fig. I schematische Zeichnungen. Normalerweise kommt ein voliständiger AusguB des Duodenums nicht vor. - I. Magen von Übergangsform (F a u l haber) zwischen Stierhorn und Hackenmagen. Normale Lage und Form des Duodenums. Winkelig besonders am peritonealen Fixationspunkt $(+)$ geknicktes Duodenum. - II. Leicht ptotischer Hackenmagen bei leichter allgemeiner Enteroptose. - III. Hochgradige Enteroptose und Ptose des Magens mit mitgesunkenen Pylorus ohne Mitsinken der Knickungsstelle des Duodenums. - IV. Hepatofixation des Pylorus mit Streckung des Duodenums.

fände. Übertrifft der Inhalt den Raum dieses Abschnittes und nimmt infolgedessen auch die zweite Hälfte der Pars superior in Anspruch, so wird der Überschuß sofort von der Peristaltik erfaßt und entführt, und das zuletzt Zugeflossene bleibt liegen.

Die eigentümliche Sonderstellung des allerersten Duodenalabschnittes, welche bereits G. S ch w a r ${ }^{1}$ ) bemerkt hat, habe ich durch die Bezeichnung B u l b u s d u od en i gekennzeichnet. Ich habe ferner mit Rücksicht auf den Umstand (vergl Fig. I-4), 
daß die vielen Varianten der Lage des Magens fast nie eine hori zontale Lage der Pars superior und inferior erlauben, die Bezeichnungen Pars superior, media, inferior duodeni, statt der gebräuchlichen (Pars horizontalis superior descendens und horizontalis inferior) vorgeschlagen. Der Bulbus duodeni umfaßt die erweiterte erste Hälfte der Pars superior. Er nimmt an der Peristaltik nur wenig Anteil, vielleicht nur durch allgemeine tonische Kontraktion. Auch darin nimmt aber der Bulbus duodeni gegenüber dem ganzen übrigen Dünndarm eine Sonderstellung ein, die ihn als Pufferraum der Antrumperistaltik, als Nachmagen erscheinen läßt.

In den übrigen Teilen des Duodenums geschieht die Inhaltsbeförderung derart, daß der Überschuß des Bulbus in Münzengröße, im Bilde unscharf begrenzt und nirgends das Duodenum ganz entfaltend und prall füllend, stoßweise in sekundenlangem Vorrücken eben noch verfolgbar ca. I dm weit befördert wird, um dann minutenlang zu ruhen. Die erste Etappe führt sie in der Regel an die Knickungsstelle zwischen Pars media und Pars inferior, die nächste in das Jejunam. Mit jedem Vorstoß verteilen sich die anfangs münzengroßen Portionen immer feiner und feiner, so daß nach einigen Jejunalschlingen gleichmäßig verteilte, stecknadelkopf- bis hanfkorngroße Partikel resultieren.

Unter diesen Umständen erscheint auch die Bedeutung der Valvulae conventis sive Kerkringii des Duodenums und Dünndarms und ihr Fehlen im Bulbus duodeni verständlich. Im Jejunum scheinen mir diese neben der Vergrößerung der sezernierenden und resorbierenden Kontaktfläche zwischen Darm und Darminhalt noch einem anderen wichtigeren Zwecke $\mathrm{zu}$ dienen, der durch die junge Kenntnis der stoßweisen Vorwärtsbewegung des Dünndarminhaltes nahegelegt wird, nämlich der $M$ is $\mathrm{ch} \mathrm{u} \mathrm{n} \mathrm{g}$ des Inhaltes mit den Sekrctendurch Wirbelbildung an den Faltenkanten. Daß durch sie, die als halbmondförmige Septen unregelmäßig verstreut quer zur Verlaufsrichtung ins Darmlumen eingesetzt sind, die motorische Arbeit gehemmt wird, scheint mir nur ein Mittel zum gleichen Zweck zu sein. Dadurch kann der Darminhalt unter um so größerem Druck an die hemmenden Septen geschleudert werden. Die vorkommenden Lageanomalien sind durch Fig. I ge- 
Die Duodenalstenose durch Füllung und Peristaltik radiologisch erkennbar. $6_{\text {I }}$

nügend gekennzeichnet und ihre Schilderung ist entbehrlich. Bezüglich des Dislokationsmechanismus ist nur für $2-4$ hinzuzufügen, daß die Dislokationen der Pars superior wohl ausschließlich durch die Verlagerung des Magens bzw. des Pylorus hervorgebracht werden, während die Lage der Pars media und inferior von der Größe des allgemeinen Innendrucks des Abdomens beherrscht werden und ihre Senkungen Folgen der Enteroptose sind, vor allem also des Sinkens des Dünndarmkonvolutes. „Die Knickungsstelle“ zwischen Pars superior und media $(+)$ bleibt dabei als relatives punktum fixum unberührt. Die Fig. 2, I-IV sind schematische Zeichnungen, normalerweise kommt ein vollkommener AusguB des Duodenums nicht vor. Die reichlichste, einige Zeit andauernde Duodenalfüllung ist durch Fig. I wiedergegeben, welche schon jetzt mit den späteren Figuren von Duodenalstenosen zu vergleichen zweckmäßig ist.

Die radiologische Symptomatologie der Duodenalstenose.

Die Literatur enthält nichts über diesen Gegenstand.

Vor 4 Jahren wurde mir nach den ersten Veröffentlichungen über die Rönt-

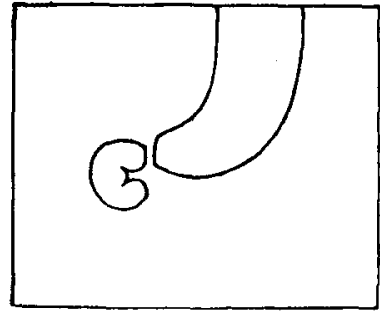

Fig. 3.

Fall I. Schirmpause des gefüllten Magens und Duodenums im Liegen. Beachte die Knickungswinkel an der Konkavität der Duodenalfüllung, welche auf den hohen Innendruck hinweisen. Über die beständig ablaufende Peristaltik desselben siehe den Text. genuntersuchung des Magens mit sichtbaren Ingesten ein I3jähriger Knabe zur Untersuchung zugewiesen, der mannigfaltige Magenbeschwerden darbot, unter denen das häufige Erbrechen ohne deutlichen Zusammenhang mit den Mahlzeiten die Hauptrolle spielte.

Das blasse, in schlechtem Ernährungszustand befindliche Kind bot objektiv nur noch eine Abweichung von der Norm, nämlich eine umfangreiche Perkussionfigur des Magens im Liegen und einen breiten und tiefstehenden kaudalen Teil des Magens nach PenzoldDehio. Es wird mit dem Verdacht auf angeborene Pylorusstenose vielleicht Duodenalstenose zur Untersuchung geschickt.

Bei der Röntgenuntersuchung füllte sich zugleich mit dem längsgestellten, hackenförmigen, in mittlerem Grade längs- und 
quergedehnten Magen das stark dilatierte und scharf gekrümmte und gespannte ${ }^{1}$ ) Duodenum (siehe Fig. 3), und zeigt fast beständige energische Formveränderungen, die im Protokoll als wurmartig und konvulsivisch bezeichnet wurden.

Die Erscheinung war so sinnfällig und verständlich, daß auf Grund der 3 Momente der prallen Füllung, der sichtbaren Peristaltik der Wand und ihrer Effektlos i $\mathrm{k}$ e i t, welche unter normalen Verhältnissen nie beobachtet wurde, eine tiefsitzende Stenosierung des Duodenums angenommen wurde, welche der Anamnese nach wahrscheinlich eine angeborene war. Es geschah dies, weil die beobachteten Erscheinungen sich vollkommen mit denen deckten, welche man auch theoretisch für die Stenosierung des Duodenums postulieren müßte: Motilitätsstörung, die bei einem Organ, dessen normale Peristaltik dafür sorgt, daß es immer nur zerstreute Inhaltsquantitäten enthält, schon durch die Füllung zum vollständig sichtbaren Ausguß gegeben ist, und sichtbare Peristaltik, die außer ihrer Existenz dadurch, daß sie dauernd sichtbar bleibt, auch ihren mindestens verringerten Effekt beweist.

Damit war sichergestellt, daß mittels der Röntgenuntersuchung bei Duodenalstenosen charakteristische Abweichungen vom normalen Verhalten nachweisbar sein können. $\mathrm{Ob}$ dieses Bild in allen oder wenigstens vielen Fällen in genügender Vollständigkeit auftritt, um praktische Bedeutung zu gewinnen, konnte nur durch weitere Erfahrung entschieden werden. In dieser Hinsicht schien insbesondere die wichtige Stenosenperistaltik ein genaues Studium zu verdienen. Ich füge deshalb hier zunächst den chronologischen 3. Fall meiner Beobachtung ein.

F a 11 2. Anna S, Io Jahre (I. XII. I909) macht den Eindruck eines etwa 7-8 jährigen Kindes, sie ist unterernährt aber von gesunder Farbe und frischem Aussehen. Die Mutter gibt an, daß sie schon als Säugling wegen Erbrechen und anderen Magenstörungen schwer fortzubringen war und nur langsam, stets hinter gleichalterigen zurückbleibend an Gewicht zunahm. Sobald das Kind gemischte Nahrung nehmen konnte, fielen der Mutter im Erbrochenen Speisereste auf, welche von früheren Mahlzeiten stammten, und einmal hat es einen

I) Die hochgradige Füllungsspannung des Organes muß aus den beiden Knickungsstellen an der Konkavität erschlossen werden. 
grünschwarzen Körper erbrochen, der sich als Kirschkern herausstellte, obwohl es seit 1/2 Jahre keine Gelegenheit hatte, Kirschen zu essen. Sie ißt sehr wenig, erbricht öfters ohne deutlichen Zusammenhang mit den Mahlzeiten und nur selten auffallend große Quantitäten. Manchmal Schmerzen in der Nabelgegend, ebenfalls ohne Zusammenhang mit dem Essen. Vor einigen Jahren stand das Kind im KarolinenKinderspital mit Gastrektasie in Behandlung. Als es vor einigen Tagen das Spital wieder aufsuchte, wurde es von Herrn Primarius Dr.K nöpfelmacher zurRöntgenuntersuchung zugewiesen.

Außer dem oben geschilderten Allgemeinzustand bot das Kind objektiv links am Hals eine kleine Drüse. Anhaltspunkte für eine tuberkulöse Affektion fehlten im Status und in der Anamnese.

Abdomen im Niveau des Thorax, nicht gespannt, nirgends druckempfindlich, keine Steifung oder Peristaltik (auch keine anamnestische). Kohlensäure-Blähung ergibt ein übergroßes undeutlich kontouriertes Blähungsbild.

Die Röntgenuntersuchung ergab folgendes:

Die sichtbaren Ingesten passierten in gewöhnlicher Weise den Ösophagus und füllten hierauf einen vertikal gestellten, etwas längs-, nicht quergedehnten Magen (tiefster Punkt 3 kindliche Querfinger

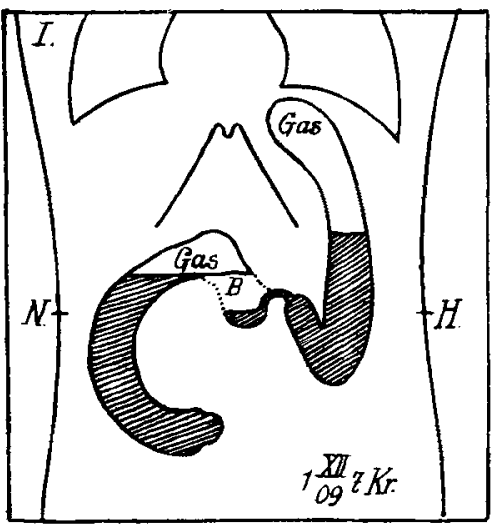

Fig. 4.

Fall 2. Schirmpause nach Einnahme der Wismutmahlzeit. Der mit seinern kaudalen Anteil nach links dislozierte Magen weist keine Querdehnung auf. Er hat den großen Teil der Mahlzeit durch den stets offen gefundenen Pylorus rasch ins Duodenum entleert, das inn nicht weiter gibt. Es ist mächtig gedehnt und in den oberen Anteilen mit Gas gefüllt. Das Bild stellt den Ruhezustand zwischen den raschen peristaltischen Formveränderungen dar, welche in Fig. 5 wiedergegeben sind. NH Nabelhorizontale.

unter dem Nabel, quere Breite am kaudalen Pol 3 Querfinger). Sein kardialer Teil liegt der Norm entsprechend unter dem medialen Anteil der linken Zwerchfellhälfte. Der kaudale Teil liegt aber nicht vertikal unter dem kardialen, sondern nach außen disloziert (siehe Fig. 4), der Pylorus (siehe unten) in der linken Mamillarlinie. MäBig tiefe rhythmische Peristaltik.

Fast zugleich mit dem Magen füllte sich aber das ihm an 
Rauminhalt mindestens gleichkommende Duodenum als dreiviertelkreisförmiges Gebilde von der Weite eines kindlichen Dickdarmes und so gelegen, daß die äußere Wand der Pars media der rechten Flanke anlag. Solcherart nahmen Magen und Duodenum zusammen die ganze quere Breite des Abdomens in der Nabelhorizontalen ( $\mathrm{NH}$, Fig. 4) ein und $3 / 4$ Teile davon entfielen auf das Duodenum, das normalerweise nur etwa $1 / 4$ dieser Strecke in Anspruch nimmt. Die Wismutfüllung des Duodenums war fast vollständig, nur der Bulbus duodeni war zum größeren Teil mit Gas gefüllt. Zwischen Gas und wismuthältigem Inhalt horizontale Grenzen, die Schüttelwellen zeigen. Distal endete die Füllung ziemlich scharf begrenzt und gleichmäßig gerundet in der Mittellinie. Darüber hinaus, insbesondere im linken unteren Bauchquadranten, fand sich auch bei längerer Beobachtung kein Wismutinhalt im Dünndarm.

Sind vollständige Füllung des Duodenums und die mangelnde Weiterbeförderung seines Inhaltes schon an sich zwei grobe Abweichungen von der Norm, so zog überdies eine beständig wiederkehrende Bewegungserscheinung den Blick auf das Füllungsbild des Duodenums. Wer den früher geschilderten Fall gesehen hatte, mußte den Eindruck haben, daß es sich um den gleichen Mechanismus handelte. Nun ging ich daran, die früher als konvulsivisch oder wurmartig bezeichneten Bewegungen zu analysieren. Es ergab sich zunächst, daß es sich um einen periodischen Vorgang handelte: Alle $6-7$ Sekunden spielte sich der gleiche, $2-3$ Sekunden dauernde Vorgang $\left.a b^{\mathbf{1}}\right)$. Eine einzelne derartige Revolution von 2-3 Sekunden Dauer verlief in einem gleichmäßigen Zuge, aus dem ich mittels rascher skizzenhafter Schirmpause nach und nach die folgenden Phasen, welche zum Verständnis des Vorganges genügen, festhielt. Jedesmal verkürzte sich der ursprünglich (Fig. 2, I) dreiviertelkreisförmige Schattenring vom oberen Ende her unter Zunahme seiner queren Breite, wie dies Fig. 3, II und III zeigen, bis er nur etwa die Hälfte seiner ursprünglichen Länge aufwies, und das Bild der ersten Hälfte vollständig verschwunden war. Dann verkürzte er sich

1) Die Messung nach dem Puls und nachherige Umrechnung, welche in der Eile angewandt wurde, macht auf absolute Genauigkeit keinen Anspruch. 
in gleicher Weise weiter, aber die erste Hälfte füllte sich rückläufig, zunächst in Form eines etwa bleistiftdünnen Bandes von 3 Querfinger Länge (5, IV), dann in voller Breite des Duodenums. Sooft dann wieder alle Duodenalanteile gefüllt waren $(5, \mathrm{~V})$, lief noch die Enge $p$ p in IV und $\mathrm{V}$ bis an das Ende der Duodenalfüllung, dann war I in Fig. 2 wiederhergestellt und eine Ruhepause von mehreren Sekunden trat ein.

Die geschilderten Erscheinungen erklären sich als effektlose peristaltische Arbeit des Duodenums und es ergibt sich aus ihnen, und zwar besonders aus der dreiquerfingerlangen Enge, die bisher nicht geläufige Tatsache, daß die Peristaltik nicht aus einem wenige Ringfaserschichten erfassenden kurzen Kontraktionsring, sondern aus einer längeren Kontraktionssäule besteht, welche in gleichbleibender Länge fortschreitet ${ }^{\mathbf{1}}$ ).

Bemerkenswert ist, $\mathrm{da} B$ der Bulbus duodeni $\mathrm{B}$ bzw. die kleine in ihm liegende Quantität wismuthältigen Inhaltes an der Peristaltik keinen Anteil nahm, ferner daß der Pylorus nicht als wismutfreier

I) Verfasser hat die gleiche Erscheinung einer Konstriktionssäule am Ösophagus bei Stenosen als Regel beobachtet.

Deutsche Zeitschrift f. Chirurgie. 105. Bd.

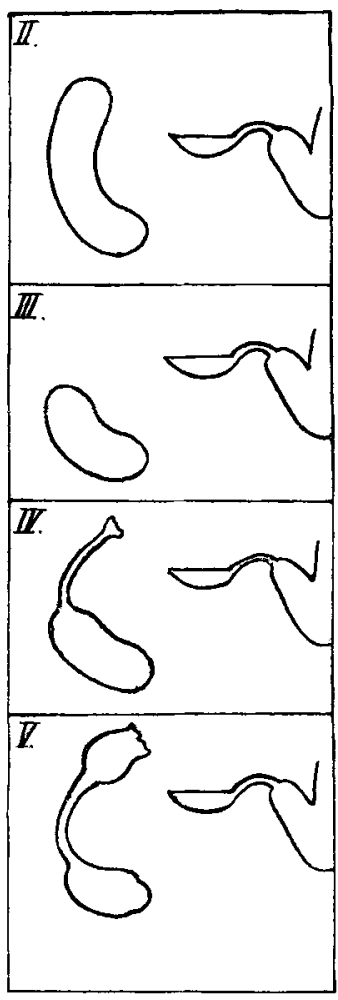

Fig. 5.

Der gleiche Fall wie in Fig. 4. Die in 2-3 Sekunden ablaufende mit Intervallen von 6 Minuten wiederkehrende Peristaltik des pathologisch gefüllten Duodenums ist hier in vier weiteren Phasen nach Schirmpausen wiedergegeben. In II und III weist die Verkürzung und Verbreiterung des Duodenums auf eine vorwärtsdrängende vollständig abschnürende Kontraktion hin. IV und $V$ zeigen, daß es sich nicht um einen Kontraktionsring, sondern um einen Kontraktionszylinder handelt, der mangels motorischen Effektes schlieBlich etwa geöffnet und rückläufig durch flossen wird. 
Spalt zwischen der Magen- und Duodenalfüllung stand, wie es der Norm entspricht, sondern beide in ca. $2 \mathrm{~mm}$ breiter Füllung miteinander verband; er war also nicht geschlossen, um sich rhythmisch zu öffnen, sondern beständig mäßig weit offen (Pylorusinkontinenz).

Bei inkontinentem Pylorus ist es einigermaßen auffällig, daß der Magen nicht durch Rückstauung dilatiert war. Doch wäre diese Erscheinung erklärlich, wenn die Rückstauung zwar vorhanden gewesen wäre, ausgedrückt etwa durch eine abnorme Verweildauer, wenn es aber zur Dehnung als einer sekundären, vielleicht einer Spätfolge der Stauung noch nicht gekommen wäre. Wir wissen ja noch nichts über die größere oder geringere Leichtigkeit, mit der die Gastrektasie bei Duodenalstenose eintritt. Überdies kann sie hier möglicherweise auch deshalb nicht in Erscheinung getreten sein, weil sich der Magen rasch und größtenteils in das Duodenum entleert hat und so nicht genügend gefüllt war.

Der Fall lehrt außer den wichtigen Details der Stenosenperistaltik des Duodenums, daß die Fälle von angeborener, vielleicht auch andere Fälle tiefsitzender Duodenalstenose bei höherem Grade der Enge das in Fall I und 2 identische Bild fast zu bieten scheinen. Wie verhalten sich nun Fälle mit höherem Sitz der Stenose?

K. S., 30 Jahre, 24. III. Igog. Zur Untersuchung wegen Magendilatation? Pylorusstenose? etwa auf Basis eines Ulcus ventriculi, zugewiesen.

Im 22. Lebensjahre hatte Patient durch 2 Tage $\mathbf{H}$ ä $\mathrm{m}$ a $\mathrm{t}$ e $\mathrm{m}$ e $\mathrm{s}$ is und längere Zeit Magenbeschwerden. I Monat war er bettlägerig. Vor 3 Jahren Drücken im Magen nach dem Essen, saures Aufstoßen, mehrmals pechschwarze breiige Stühle, kein Erbrechen.

Seit einem Monat Magendrücken und einige Stunden nach dem Essen fast regelmäßig Erbrechen, oft alter $\mathrm{S}$ p e is en. A b$\mathrm{m}$ a g e ru ng. Ob Galle im Erbrochenen war, ließ sich nicht sicher ermitteln.

Die Durchleuchtung ergab einen stark längs- und in leichtem Grade quergedehnten Magen (tiefster Punkt im Stehen 4 Querfinger unter dem Nabel, größte quere Breite $4^{1 / 2}$ Querfinger $^{1}$ ).

I) Eine so geringe Ausdehnung kommt bei Hackenmagen auch als atomische Weite vor. 
Die Duodenalstenose durch Füllung und Peristaltik radiologisch erkennbar. 67

Die Vollständigkeit seiner Füllung, seine palpatorische Inhaltsverschieblichkeit und seine Peristaltik, sowie sein sonstiges Verhalten boten nichts, was auf eine grob anatomische Wandveränderung hingewiesen hätte.

Außer dem Magen füllte sich auch die Pars superior duodeni. Er war vom Magen durch den meist offenstehenden (mit gefüllten sichtbaren Lumen ausgestatteten) Pylorus getrennt.

Hier muß bemerkt werden, daß das bei Beginn der Durchleuchtung sichtbare Bild (Fig. 6) nicht erlaubt hätte, den am weitesten rechts gelegenen $\mathrm{Ab}$ schnitt der Füllung als Durodenalfüllung zu agnoszieren. $\mathrm{Er}$ konnte auch noch dem Magen angehören und dann wäre es nur nötig gewesen, in der Pars pylorica Einschnürungen (Ulcus, Narbe, Spasmus usw.) anzunehmen. Diese Frage, die, falls etwa nur das Photogramm vorliegt, unlösbar ist, ließ sich bei der Durchleuchtung, dadurch erledigen, daß die Antrumperistaltik den Ort des Pylorus deklarierte. Auch unser Magen zeigte, daß die Abschnürung (Fig. 6 Sph.) durch den Sphinkter antri hervorgerufen war, indem der distal von ihm gelegene kugelige Abschnitt sich konzentrisch kontrahierte, dadurch verschwand und hierauf ohne die

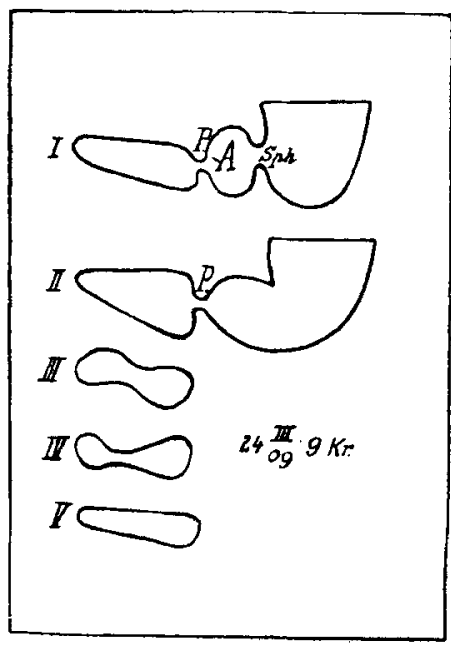

Fig. 6.

Fall 3. Stenose am Übergang zwischen Pars superior und Media duodem. In I zeigt die typische Antrumperistaltik $(\mathrm{A}=$ Antrum, Sph. $=$ Sphinktu antri, $\mathrm{P}=$ = Pylorus), daß der Endteil der Füllung das Duodenum ist. II Anreicherung der Duodenalfüllung durch die Antrumperistaltik. III-V Peristaltische Formveränderungen einer Revolution. Abschnürung Sph. sich wieder füllte (Fig. 6, II), und diese Veränderung rhythmsich ca. alle 2I Sek. erfolgte. Dadurch war A als Antrum, $\mathbf{P}$ als Pylorus und das fragliche Gebilde als Duodenum sicher gestellt. Mit jeder Antrumkontraktion nahm der Duodenalinhalt an Menge zu.

Die Füllung des ersten Duodenalabschnittes wäre keine Abweichung von der Norm gewesen, wenn sie nur den Bulbus duodeni und nicht die ganze Pars superior betroffen hätte, und nicht prall und dauernd gewesen wäre (siehe die Einleitung). Dazu 
kam nun noch als wichtigstes Symptom, daß auch dieser Fall während der ganzen Beobachtungszeit (insgesamt I5 Minuten an zwei verschiedenen Tagen) $\mathrm{rhyth-}$ mische Formveränderungen peristaltischen Ursprunges zeigte, aus denen $I$ bis 5 in Fig. 6 einzelne festgehaltene Phasen wiedergeben. Während der ganzen Beobachtungszeit wurde das übrige Duodenum und das Jejunum von Ingesten durchflossen, und diese füllen in einer gegen die Norm nicht zurückbleibenden Menge den Dünndarm. Die Motilitätsstörung erscheint also für Brei kompensiert, sie ist nur örtlich insofern vorhanden, als ein kurzer Abschnitt im $6 \mathrm{~m}$ langen Dünndarm eine für die Gesamtdurchtreibungszeit nicht in Betracht kommende Verzögerung aufweist, so daß es abnormerweise zur prallen Füllung kommt. Ein Rest im Duodenum etwa nach 24 Stunden war nicht zu erwarten und wurde daher nicht erhoben.

Am 3. Tage nach der ersten Untersuchung war das Colon noch mit Wismutingesten gefüllt, und dieser Umstand gab Gelegenheit zu konstatieren, daß das Quercolon am Úbergang vom ersten zum zweiten Drittel, also dort, wo es in der Nähe des Duodenums gelegen war, am neuerlich gefüllten Duodenum (mittelbar oder unmittelbar) adhärent war: es ließ sich manuell mit ihm nach oben rechts und links, aber nicht ohne das Duodenum dislozieren, und die zwischen ihnen eingedrückte Kante der Hand vermochte nicht, sie zu trennen. Die Adhäsion erklärt sich ungezwungen als eine der Duodenalstenose koordinierte peritonitische Konsequenz des Ulcus, auf das die Blutungen hinweisen. Die Wiederholung der Untersuchung des Duodenums ergab den gleichen Befund. Der Befund wurde in diesem Sinne mit der der Neuheit des Bildes entsprechenden Reserve abgegeben. Der Chirurg stellte das Freisein des Pylorus fest, führte die Gastroenteroanastomose aus, hatte aber nicht Gelegenheit, die Durchgängigkeit des Duodenums zu prüfen.

Die Besonderheiten des Falles sind der hohe Sitz der Stenose, so daß nur ein kurzes Stück des Duodenums im Stauungsgebiet liegt und die Dilatation des Magens, von der wir schon sahen, daß sie nicht konstant, ja nicht einmal häufig ist. Es liegt nahe, zwischen diesen beiden Momenten einen inneren Zusammenhang zu vermuten. 
Trotzdem die Stenose eine relativ gute Durchlässigkeit zeigte, war doch eine Dilatation des Magens aufgetreten, vielleicht weil das im Stauungsgebiet liegende Stück sehr klein war und trotz Dehnung nur einen geringen Teil des Mageninhaltes aufnehmen konnte. Die motorische Arbeit hinter der Stenose mußte also das Duodenum und das Antrum pylori leisten, und beide begannen wohl $\mathrm{zu}$ erlahmen. In Fall 2 kann das Duodenum vermöge seiner Weite den ganzen Mageninhalt aufnehmen, und so kam es hier nicht zur Dilatation.

Verglichen mit den zwei ersten Fällen zeigt der neue zwar wesentliche Differenzen, dieselben können aber auf den höheren Sitz zurückgeführt werden. Dagegen scheinen die konstante pralle Füllung und die ununterbrochene sichtbare rhythmische Peristaltik sich als durchgreifende Symptome zu bewähren. Eine ausgesprochene Herabsetzung der motorischen Förderung in dem Sinne, daß auffallend wenig in das Jejunum geschafft wird, war nicht zu konstatieren. Die im Jejunum auftretenden Mengen waren sogar recht erhebliche. Eine derart a uffallende M otilitäts stör u ng, daß man, wie in den früheren Fällen von effektloser oder effektarmer Peristaltik sprechen kann, m u B a lso nicht vorhanden sein, wie das ja begreiflich ist bei geringgradiger Stenose und großer Muskelkraft hinter der Enge. Wichtig aber ist, daß der Fall zeigt, daß auch ohne nennenswerte Motilitätsstörung die pralle Füllung und die Peristaltik zustande kommen und die Stenose verraten. Übrigens wurde nicht festgestellt, ob das Duodenum nicht nach abnorm langer Zeit noch Inhaltsreste aufwies. Dieser Punkt wurde im nächsten Fall beachtet.

F a 11 4. J. A., 54 Jahre, Pr. 25. VIII. I909, 8 von der I. medizin. Abteil. zur Untersuchung des Magens zugewiesen. Patient, der früher stets gesund war, leidet seit 3 Jahren an Magendrücken, das unabhängig vom Essen auftritt, wenn auch häufiger bei leerem Magen. Manchmal erbrach er auch. Seit 5 Monaten steigerte sich das Druckgefühl zu h eftigen Schmerzen, die gewöhniich $I^{1 / 2} \mathrm{St} u n d$ e $n \mathrm{nach}$ dem Essen auftraten und a uf deren $H$ öhepunkt Erbrechen sehr groBer Mengen Mageninhaltes erfolgte. Blut wurde nie erbrochen. Der Appetit war gut. Der somatische Befund war negativ; im Probefrühstïck fand sich geringe $\mathrm{H}$ y p e r a $\mathrm{z}$ i d i t ä t. 45/65; keine Magensteifung, kein Rückstand. 
Die Röntgenuntersuchung war auf den Magen gerichtet. Ich gebe sie ausführlich wieder, weil auch ihre einzelnen Phasen wie sich zeigen wird, für den Gegenstand bedeutungsvoll sind. Sie wurde von einem meiner Assistenten begonnen. Diesem fiel bei der Voruntersuchung mit der Wismutwasseraufschwemmung eine unverständliche Erscheinung auf, darin bestehend, daß nach Auftreten des in Fig. 7 wiedergegebenen Bildes der säckchenförmige Teil B rhythmische Bewegungen abwechselnd nach oben und nach der rechten Seite hin ausführte. Er zog mich zur Untersuchung zu und ich stellte zunächst fest, daß der Behälter der

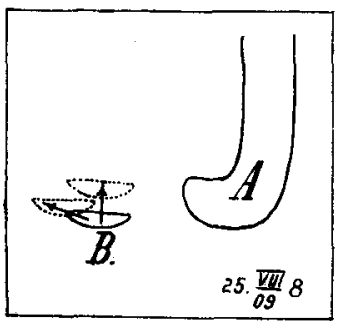

Fig. 7 .

Fall 4. Füllungsbild des Magens und Duodenum bei Stenose in der Mitte der Pars superior, sofort nach Wismutwasseraufschwemmung. Die Duodenalfüllung scheint sich ohne Formveränderung rhythmisch nach oben und rechts zu bewegen. fraglichen Wismutmenge, welcher den Eindruck eines abgeschnürten Teiles des Magens machte, $\mathrm{nich} t$ e $t$ wa respiratorisch bewegt wurde, wie andere Bauchorgane dies häufig werden (und zwar desto ausgiebiger, je näher sie dem Motor der Bewegung, dem Zwerchfell liegen). Respiratorische Bewegung zeigte beispielsweise die Magenfüllung $A$; auch lie $B$ sich mit der Hand am Thorax feststellen, daß die sichtbarere Bewegung mit der Atmung nicht synchron war. Die Bewegung war auch nicht pulsatorisch (Aorta), sie war viel zu selten und arhythmisch.

Also mußte sie einer Peristaltik ihren Ursprung verdanken. Obwohl sie sich in Zeiträumen einstellte, welche ungefähr der Peristaltik des Magens, insbesondere des Antrum pylori, entsprach $^{1}$ ), konnte doch $\mathrm{nicht}$ an eine Antrumperistaltik gedacht werden, weil nur Verschiebung, nicht Verkleinerung ${ }^{2}$ ) eintrat. So blieb außer dem D u od e n $\mathbf{u}$ m,

I) Zirka 25 Sekunden.

2) Auch bei stenosiertem Pylorus kontrahiert sich das Antrum bis zum Verschwinden seines Inhaltes. Dieser wird wie bei der Mischperistaltik rückwärts in den Magen entleert. 
dessen Stenosierung die Erscheinung als Stenosenperistaltik gut erklären konnte, noch ein anderer Teil des Dünndarmes und der Dickdarm möglich, aber diese zwangen außer der nötigen Kommunikationsfistel mit Magen oder Duodenum zur weiteren Annahme einer Stenose in dem betreffenden Darmabschnitt unterhalb der Fistel, was die letzteren Annahmen sehr kompliziert erscheinen läßt. Die Gründe, welche für das Duodenum sprachen, waren, daß der Behälter nicht der Magen war, daß er sich sofort nach dem Magen füllte, daß der Rhythmus der Bewegung mit dem mir von den früheren Fällen bekannten ungefähr übereinstimmte, und daB diese Annahme die klinischen Erscheinungen, die Magenektasie bzw. das kopiöse Erbrechen erklärten. Das spezielle Bild ließ sich dadurch erklären, daß das Duodenum neben älterem Inhalt zu unterst Wismut enthielt, so daß nur die Bewegungen eines kleinen Wandabschnittes, der unteren Wand des Bulbus duodeni, zur Beobachtung kam. Uner-

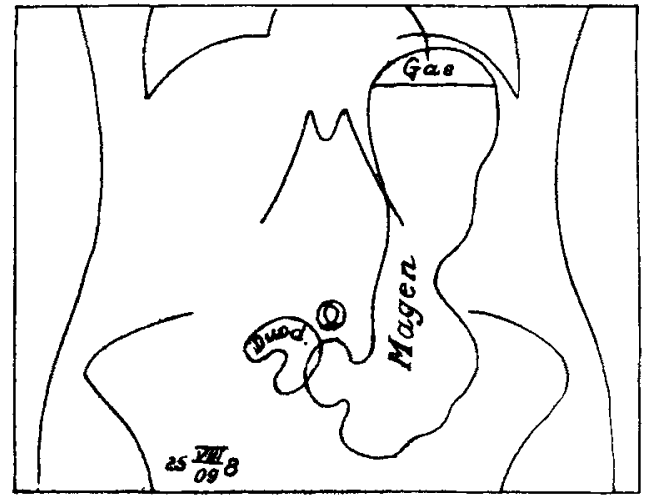

Fig. 8.

Der gleiche Fall. Wismutmahlzeit. Erhebliche Längs- und Querdehnung des Magens und ausgußartige Füllung des ersten Duodenalabschnittes, eine der thythmischen peristaltischen Formverändcrungen zeigend.

klärt blieb, daß die Ortsveränderung bald nach oben, bald nach außen gerichtet war.

Nun wurde der Magen gefüllt. Er erwies sich als stark längsund quergedehnt (handbreit unter dem Nabel lag im Stehen der tiefste Punkt, 6 Querfinger maß die quere Breite des kaudalen Poles). Die Peristaltik begann hoch ${ }^{1}$ ) und zeigte Kontraktion, welche weit vor dem Sphinkter antri den Magen vollständig quer abschnürten ${ }^{2}$ ). Vollständige und regelmäßige Antrumperi-

I) An der Pars cardiaca, normal an der Pars pylorica.

2) Normal erst am Sphinkter antr. 
staltik zeigte den Endteil, der Pylorusspalt den Endpunkt des Magens an. Er wurde nach rechts von einem über walnußgroßen, prall gefüllten Bulbus duodeni begrenzt. Dieser erfuhr in Intervallen, welche denen der Antrumperistaltik entsprachen ${ }^{1}$ ), kurz dauernde Formveränderungen, von denen Fig. 9 einzelne Phasen wiedergibt ${ }^{2}$ ).

Nach etwa $1 / 4$ stündiger Beobachtung waren trotz der imposanten peristaltischen Arbeit des Duodenums keine sichtbaren Mengen in den weiteren Dünndarmschlingen angelangt und 24 Stunden später war zwar die Hauptmasse des Wismut im Colon ascendens, Cöcum nur in den letzten Ileumschlingen, aber im Bulbus duodenilag noch ein ihn zur

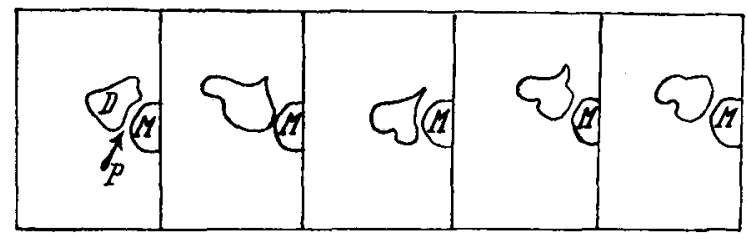

Fig. 9 .

Der gleiche Fall. Andere Phasen der rhythmischen Formveränderung des gefüllten prästenotischen Duodenalabschnittes.

$\mathrm{H} \ddot{\mathrm{alfte}} \mathrm{f} \ddot{\mathrm{l}} \mathrm{lle} \mathrm{nder} \mathrm{R}$ est und zeigte die gleichen Erscheinungen wie im Beginn der Untersuchung (Fig. 9).

Res üm e e: Schon bei der Voruntersuchung im höchsten Grade wahrscheinlich, konnte die Duodenalstenose bei der Hauptuntersuchung bezüglich ihrer Lokalisation und bei der 24 stündigen Nachuntersuchung bezüglich ihres Grades genau bestimmt werden. Die Hauptsymptome waren: Vollständige Füllung des ersten Duodenalabschnittes, effektarme Peristaltik, 24 stündige Austreibungszeit, Dilatation des Magens.

Der Fall, der einen noch höheren Sitz als der vorhergehende aufwies, scheint mir deshalb von besonderer Wichtigkeit zu sein, weil er zeigt, wie geringfügig die Erscheinungen sein können,

I) $\mathrm{Ob}$ Synchronismus bestand, wurde leider nicht festgestellt.

2) Eine regelmäßige Aufeinanderfolge seitens der verschiedenen Formen konnte nicht festgestellt werden. Daher konnten auch die beobachteten wellenartigen Bewegungen keine Deutung finden. 
die die Diagnose erlauben, wenn man sich an die aus den früheren Fällen gewonnenen Abstraktionen hält. Wenn der Mageninhalt einen weiteren Raum mit scharfer Konturierung füllt und dieser rhythmische peristaltische Aktionen zeigt, so ist die Duodenalstenose auch dann im höchsten Maße wahrscheinlich gemacht, wenn der gefüllte Raum zu klein ist, um aus seiner Form als Duodenum oder Teil desselben erkennbar zu sein. Man kann dann insbesondere noch in Zweifel sein, ob der gefüllte Raum Pars superior oder inferior ist. Die vollständige Füllung des Magens beantwortet die Frage durch die Lagebezeichnung zum Pylorus.

In unserem Fall bestätigte die Fortsetzung der Untersuchung, daß die auf der ersten geringfügigen Erscheinung aufgebaute Annahme richtig war. Solcherart scheint schon die gewöhnliche Magendurchleuchtung in ihrer ersten, bloß ein halbes Glas Wasser verwendenden Voruntersuchung uns bei dem ersten Blick auf den Schirm manchmal alles zu bieten, was zur Feststellung des Krankheitsbildes notwendig ist, jedenfalls aber etwas, was durch die Bewegungserscheinung, die dazu gehört, sinnfällig ist und bei einiger Aufmerksamkeit darauf hinlenkt, alles zur Feststellung Nötige (stärkere Füllung durch Effleurage oder weitere Aufnahme von wismuthaltigen Ingesten) $\mathrm{zu}$ veranlassen und $\mathrm{zu}$ beachten. Die Ermittlung der Duodenalstenose muß daher, wenn man von ihren radiologischen Symptomen weiß, geradezu als einfach und leicht bezeichnet werden.

Der Fall zeigt ferner, wie bei hochgradiger Stenose oder erlahmter Vis a tergo die $\mathrm{M}$ agendilatation, der a ugenscheinlich geringe Effekt an Jejunalfüllung und der späte Rest im Duodenum parallel gehen, indem hier alle 3 Momente gleichmäßig stark ausgeprägt waren, während sie im vorausgehenden fast vollständig fehlten.

Fall 5. W. P., 40 Jahre, Pr. 20 X. Igog sei, um Wiederholungen zu vermeiden, nur in den Hauptzügen geschildert: T i e f e r S it z (U்bergang vom Duodenum zum Jejunum), wie in den beiden ersten Fällen von angeborener Stenose, und dabei $A$ uf $t$ rete $n$ in späteren Lebens jahren. Ferner war hinter der Enge nicht das ganze Duodenum prall gefüllt, sondern bloß die Pars inferior und die zweite Hälfte der Pars media. Keine sichere Querdehnung des Magens. 
Die Peristaltik zeigte nicht eine Kontraktionssäule, sondern einen kurzen Kontraktionsring. Sonst glich der Fall den anderen, besonders dem Fall 2.

Radiologische Semiologie der Duodenalst e nose.

Aus den geschilderten Fällen im Zusammenhang mit dem physiologischen Verhalten ergibt sich folgende Bewertung der radiologischen Symptomatologie der Duodenalstenose:

Die anatomische Verengerung selbst kann nicht wahrgenommen werden; der Nachweis stützt sich vielmehr auf die motorischen Folgeerscheinungen; daher ist er nur bei funktionell in Betracht kommenden Engen möglich. Leichte Einschnürungen, welche den Namen einer Stenose nur im weiteren Sinne verdienen und funktionell ohne alle Konsequenzen sind, können nicht nachgewiesen werden $\left.^{\mathbf{1}}\right)$. Funktionell in Betracht kommende Engen zeigen:

Ausfüllung des Duodenum hinter der Stenose, so daß die Wand in scharfer Begrenzung sicht$\mathrm{b}$ a $\mathrm{r}$ ist. Während normalerweise nur zirka münzengroße Qualitäten von unscharfen Konturen während der peristaltischen Fortschaffung also in Bewegung oder zwischen zwei peristaltischen Akten in Ruhe sichtbar sind, füllt sich hier das Lumen des Duodenum hinter der Stenose, so daß die Wände desselben dem Ausguß scharfe Grenzen verleihen und ihn meist wurstartig gekrümmt gestalten. Seine Länge hängt ab vom Sitz der Stenose (desto länger, je tiefer), vom Grad der Mobilitätsstörung (desto länger, je hochgradiger die Enge und je schwächer die Vis a tergo, die vom Duodenum und vom Magen beigestellt wird), endlich von der Menge des den Magen verlassenden Ingesten. Wird das letztere Moment durch reichliche Aufnahme und Effleurage der wismuthaltigen Ingesten aus dem Magen ausgeschlossen, so kommt dem hohen Sitz natürlicherweise, den Tiefen nur dann eine kurze Füllungssäule zu, wenn die Enge relativ leicht überwunden wird.

Eine, wie es scheint, neben Magenatonie vorkommende atonische Motilitätsstörung im Duodenum, insbesondere dann,

I) Außer als zweite Engen vor einer groben Stenose. Im Magen sind bekanntlich auch relative Engen sichtbar. 
Die Duodenalstenose durch Füllung und Peristaltik radiologisch erkennbar. 75

wenn die Zufuhr von Mageninhalt eine reichliche ist (z. B. Insuffizienz des Pylorus), zeigt sich dadurch, daB in der Pars in ferior einige Inhaltsportionen aneinander gereiht liegen bleiben. Sie zeigen aber, offenbar, da sie nicht unter dem Nachdruck der Peristaltik stehen und das Duodenum nicht ganz entfalten, keine scharfe Wandkontur. Überdies bieten sie keine peristaltischen Formveränderungen dar und können nur durch die nächste Peristaltik die normale Ortsveränderung erfahren. Diese Störung kann darum mit dem Ausguß bei Stenosen nicht verwechselt werden.

Der StenosenausguB ist daher die durch die Menge des gestauten Inhaltes und durch die peristaltische Vis a tergo entstehende, die Wände vollständig entfaltende Füllung der hinter der Stenose liegenden Duodenums.

Die Stenosenperistaltik des Duodenalausgusses kann mit der normalen Verschiebung der Inhaltsportionen nicht verwechselt werden. Die normale zeigt nur den Effekt der Verschiebung des Inhaltes, jene macht im Gegenteil den Eindruck einer völlig effektlosen, rhythmisch wiederkehrenden Formveränderung.

Was man normalerweise sieht, ist eine Ortsveränderung, was man hier sieht, ist eine Formveränderung. Sie muß trotzdem nicht wirklich effektlos sein, weil die oft kaum sichtbaren ausgetriebenen Mengen durch schwersichtbare vom Magen neu hinzukommende ersetzt werden.

Die Stenosenperistaltik ist ein konstantes Symptom, sie beginnt sobald, und dauert solange als Inhalt im Duodenum ist, siebetrifft a 1 l e F äll e. Sie durchläuft in einigen Sekunden das Duodenum und wiederholt sich rythmisch in Intervallen von ca. 7 Sekunden. Die Intervallen waren bei 3 von 4 daraufhin beachteten Fällen ungefähr die gleichen, nur in einem gibt das Protokoll noch längere Intervallen an. Doch muß dieser Punkt, da keine exakten Vorrichtungen zur Zeitmessung im Dunkeln vorgesehen waren, noch eingehender studiert werden.

Die K o n stan z der Peristal t ik, in allen Fällen und 
während der ganzen Beobachtungszeit, die für die Aufdeckung der Stenose so wertvoll ist, hat keine Analogien am Magen und am Dickdarm, wo die Stenosenperistaltik nicht unausgesetzt andauert. Im Duodenum dürfte sie daher rühren, daß für dieses nur eine ungemein kurze Verweildauer der Ingesten präformiert ist. Wir haben es daher nicht mit zwei gesondert zu erhebenden Symptomen zu tun, dem Stenosenausguß und der Stenosenperistaltik, es treten vielmehr beide zugleich auf: $W$ e $n \mathrm{n} A$ us $\mathrm{g} \mathrm{u} B$, $\mathrm{d}$ a n $\mathrm{n}$ a u $\mathrm{ch} \mathrm{Per}$ istaltik und dieses auffallende Syndrom garantiert die Häufigkeit der Feststellung.

Der Ausguß ist die einzige Mobilitätsstörung, welche in den erkennbaren Fällen bestehen muß, und er bedeutet den geringsten Grad einer solchen, wenn er allein besteht. Man sieht dann im Jejunum bald Chymusmengen auftreten, welche von den normalen nicht sicher abweichen.

Höhere Grade zeigen kein e de ut 1 ich en Chymus m e $\mathrm{n}$ g e $\mathrm{n}$ i m J e j u n u m, was besonders auffalledner ist, wenn im Duodenum noch peristaltisch stark bearbeiteter Inhalt liegt. Im Duodenum gibt es keine physiologische Rückbewegung wie im Antrum ventriculi, daher bedeutet jedes Mißverhältnis zwischen Duodenuminhalt und -peristaltik einerseits und Jejunalinhalt anderersseits eine Motilitätsstörung, deren Grad dem Grad des Mißverhältnisses gleich ist.

Z wei weitere nachweisbare Konsequenzen höherer Grade der Duodenalstenose sind das zeitliche Zurückbleiben der Förderarbeit gegenüber der Norm, also d a s A uftreten von Rest en, ferner das Entstehen einer Erlahmungsdilat ation hinter der Stenose. Diese betreffen sowohl das Duodenum als den Magen. Es müssen nicht beide in gleicher Weise betroffen sein. Reste im Duodenum allein wurden im Fall 4 und 2, Dehnung des Duodenum allein Fall 2 gefunden. Magen und D u ode $\mathrm{num}$ werden um sogleichmäBiger betroffen, je höh er die Stenose, umso ungleichmäBiger, je tiefer sie sitzt, weil im letzteren Fall das gedehnte Duodenum in seiner ganzen Länge eine Kapazität haben kann, welche gegenüber der des Magens nicht zu vernachlässigen ist und diesen daher 
Die Duodenalstenose durch Füllung und Peristaltik radiologisch erkennbar. 77

nach Vollendung seiner motorischen Arbeit mehr minder weitgehend, im extremsten Falle vollständig entlasten kann.

Das letztere Verhalten war im Fall 2 vorhanden, wo die Kapazität des Duodenum der des Magens nicht nachstand. Wenn Magen und Duodenum bezüglich der Reste und der Dilatation nicht parallel gehen, dann ist naturgemäß das Duodenum mehr als der Magen betroffen, weil die Rückstauung hinter der Stenose dieses in erster Linie und schon bei kleinen Inhaltsquantitäten und geringem Grad der Enge betrifft.

Bezüglich der Dehnungsmasse des Magens verweise ich auf die diesbezügliche Literatur. Sie kann die höchsten Grade erreichen (Fall 4). Die D e h n ung des Magens ist bei hohem Sitz häufig, freilich erst im Dekompensationsstadium, b e i tief e m Sit $z$ der Duodenalstenose scheint sie aber selten respektive sehr spät einzutreten, so daß die mangelnde Dehnung des Magens nicht wie bisher gegen Duodenalstenose verwendet werden darf.

Hier möge noch die Bemerkung Platz finden, daß der Möglichkeit einer Kombination von Pylorus und Duodenalstenose deshalb Beachtung geschenkt werden soll, weil das Ulcus duodeni auf Basis der gleichen Anomalie zu entstehen scheint wie das häufigere des Magens, so daß es wahrscheinlich ist, daß die meisten Träger eines solchen auch ein Ulcus ventriculi erlebt haben.

Das Duodenum kann auf 2 und $\mathbf{2}^{\mathbf{1} / 2}$ Querfinger Durchmesser und mehr gedehnt werden.

Bezüglich der R es t e können die gleichen normalen Zahlen für beide gelten, die bisher für den Magen in Betracht kamen, also 6 Stunden als Maximum, was darüber ist, ist Motilitätsstörung, wobei einige Stunden mehr als leichter Grad zu deuten sind, dann mittlerer und von etwa 24 Stunden schwerer angenommen werden muß, auch wenn im letzteren Fall dazwischen Mahlzeiten eingenommen werden.

Schließlich muß bemerkt werden, daß es nach den bisherigen Erfahrungen bezüglich spastischer Kontraktions $z$ ustände am Verdauungsrohr sehr wahrscheinlich ist, daß der meist sekundäre, durch Ulcerationen ausgelöste Spasmus genau die gleichen Erscheinungen hervorrufen kann, wie die Stenose. Warum sollte auch die in der Dauer genügend unbe- 
schränkte funktionelle Stenose andere Konsequenzen haben als die anatomische. Wenn daher die Stenose nachgewiesen ist, hat man nach Zeichen der Floridität einen eventuellen Ulcus (occulte Blutung!) und nach anamnestischem Wechsel der Beschwerden von Null bis zur vollen Höhe zu forschen und diesfalls die Untersuchung nach entsprechender Zeit zu wiederholen. Der Spasmus ist sicher viel häufiger als bisher angenommen wurde.

Untersuchungsmethode.

Da der Anlaß, auf Duodenalstenose zu prüfen, selten durch die anderen Untersuchungsresultate gegeben ist, sondern fast stets bei der Magenuntersuchung durch das eine oder andere zufällig bemerkte Duodenalsymptom entsteht, so ist von einer von vornherein auf Duodenalstenose gerichteten Untersuchung meist nicht die Rede. Trotzdem ist es nützlich, sich klar zu sein, bei welcher Technik die Chance, sie zu erkennen, am größten ist. Der Duodenalstenose ist ja jeder Fall mit Symptomen der Pylorusstenose ferner jeder Fall mit unklaren aber schwereren Magenerscheinungen verdächtig. Daraus ergibt sich, daß wir bei der Magenuntersuchung stets das Duodenum beachten müssen. Sch h i n b a r effektlose Peristaltikamentfaltetgefüllten $\mathrm{D}$ u o de $\mathrm{n} \mathrm{u} \mathrm{m}$ ist ja ohnedies leichter zu bemerken als zu übersehen, man muß also nur wissen, daß das Organ einen Blick verdient. Schöpft man Verdacht, dann ist es zweckmäßig, wenn noch möglich, sofort sonst tags darauf sich an Folgendes zu halten: $\mathrm{N}$ ü chtern (offener Pylorus) $\mathrm{g}$ ro $\mathrm{Be} \mathrm{M}$ eng en Wis mut. wasseraufschwemmung ins Duodenum effleurieren. Sonst kann der Pylorus schon geschlossen sein, die wismuthaltige Speise kann ihm durch den erregten Sazlsäurereiz zum Schluß bringen, die Wismutsättigung der Speise kann nicht so groß sein, wie die der Aufschwemmung. Die spätere Betrachtung läßt oft nicht mehr feststellen, mit welcher Dünndarmschlinge es der Untersucher zu tun hat.

Die Ermitltung des Dehnungszustandes und der Reste in Magen und Duodenum geschieht mittelst der Wismutmahlzeit.

Die Restbestimmung im Duodenum kann praktisch dadurch vereinfacht werden, daß man verdünnte und wismutreiche 
Die Duodenalstenose durch Füllung und Peristaltik radiologisch erkennbar. 79

Mahlzeit in kleiner Quantität nehmen läßt, diese aus dem Magen ganz oder fast ganz in das Duodenum effleuriert und nun bestimmt, in welcher Zeit das Duodenum wieder leer ist. Normalerweise sind hierfür bei nachweisbarer Peristaltik ${ }^{1}$ ) wohl einige Minuten reichlich bemessen.

Am unzweckmäBigsten ist die bloße Verwendung der Photographie, doch kann sie gelegentlich einmal Beweisendes liefern. Ihr Wert liegt hier im Schaffen von Demonstrationsobjekten. R ö $\mathrm{ntg}$ e $\mathrm{nk}$ in e mato graphie ist hier wie sonst ein mehr minder guter Ersatz des Erlebens. Über die Durchleuchtung hinaus kann sie angesichts der Langsamkeit der in Betracht kommenden Bewegungsvorgänge nichts bringen.

I) Vgl. Atonie des Duodenum, S. 74 . 